\title{
Raman nicroscopic markers for genetic and other diseases of bone
}

\author{
Michael D. Morris and Tso-ching Chen
}

Department of Chemistry, University of Michigan, Ann Arbor,MI 48109

Bone tissue is a composite material consisting of carbonated apatite crystals (usually called mineral in the bone tissue literature) deposited on a matrix that is largely composed of type I collagen, with about $10 \%$ other proteins and glycoproteins. The Raman spectrum of bone is rich with signatures for both mineral and matrix. In this paper we will show how Raman microspectroscopy and and imaging can be used to study diseased tissue and also how these methodologies can be used to probe the the mechanical properties of bone at the ultrastructural (molecular) level. As with most other tissues, bone fluorescence is easily excited with green lasers, so that Raman spectra are most conveniently excited in the near infrared.

A typical bone tissue Raman spectrum is shown as Figure 1a. Characteristic bands of mineral and matrix are labeled. As bone tissue matures the mineral becomes more heavily carbonated and the $1070 \mathrm{~cm}^{-1} / 958 \mathrm{~cm}^{-1}$ intensity ratio increases. Some substitution of carbonate for phosphate is observed even in the earliest mineral formed prenatally [1]. The normal process of bone tissue development can be followed by microspectroscopy. Additionally, there are Raman markers for mechanical damage to both bone mineral and bone matrix [2]. Depending on the conditions mechanical stress can result in reversible and irreversible changes.

Genetic, metabolic and other diseases of bones may cause two kinds of spectroscopically observable changes. First, there can be an alteration in the ratio of bone mineral to bone matrix. Second, there can be changes in the chemical structure of mineral, matrix or both. Changes in mineral/matrix ratio are observable as changes in band ratios without changes in band positions. Structural changes cause changes in the Raman shifts as well as the intensities of mineral and matrix bands. We will present spectra and images showing both classes of alterations, including cases where both classes of change are observed in the same specimen.

We will discuss ultrastructural changes observed in the femoral cortical tissue of BRTL mice. BRTL is a genetically modified mouse that has a defect in Col 1 , a gene that codes for the $\alpha-1$ chain of collagen and is a model for osteogenesis imperfecta, type IV. OI is the most common genetic defect of bone tissue. The mutation causes a substitution of cysteine for glycine, preventing complete formation of the collagen triple helix. In Figure 1 we show Raman spectra of tissue from the femur of a wild type mouse (1a) and one with single (1b) and double mutations (1c). In single mutations one $\alpha-1$ chain is substituted and in the double mutation both chains are defective. The mineral bands in the spectra from the modified mouse tissue appear to be quite similar to those of the control, although the mineral/matrix ratio is reduced. Amide I and amide III are different in BRTL and in the wild type mice, indicating the presence of unwound collagen. Although the Raman spectra are different from those of normal mice, the defects are distributed uniformly across a section of tissue, as shown in the image in Figure 2.

[1] C.P. Tarnowski et al., J. Bone Miner. Res. 17 (2002) 1118.

[2] A. Carden et al., Calcif. Tiss. Internat. 72 (2003) due February issue. 
Figure 1. Raman spectra of normal murine cortical bone tissue. (a) Wild-type. (b) Single mutation. (c) Double mutation. The mineral/matrix ratio differs among the specimens. Collagen defects are observed as changes in the amide I and amide III vibrations. Inset: magnified $1100-1700 \mathrm{~cm}^{-1}$ region.

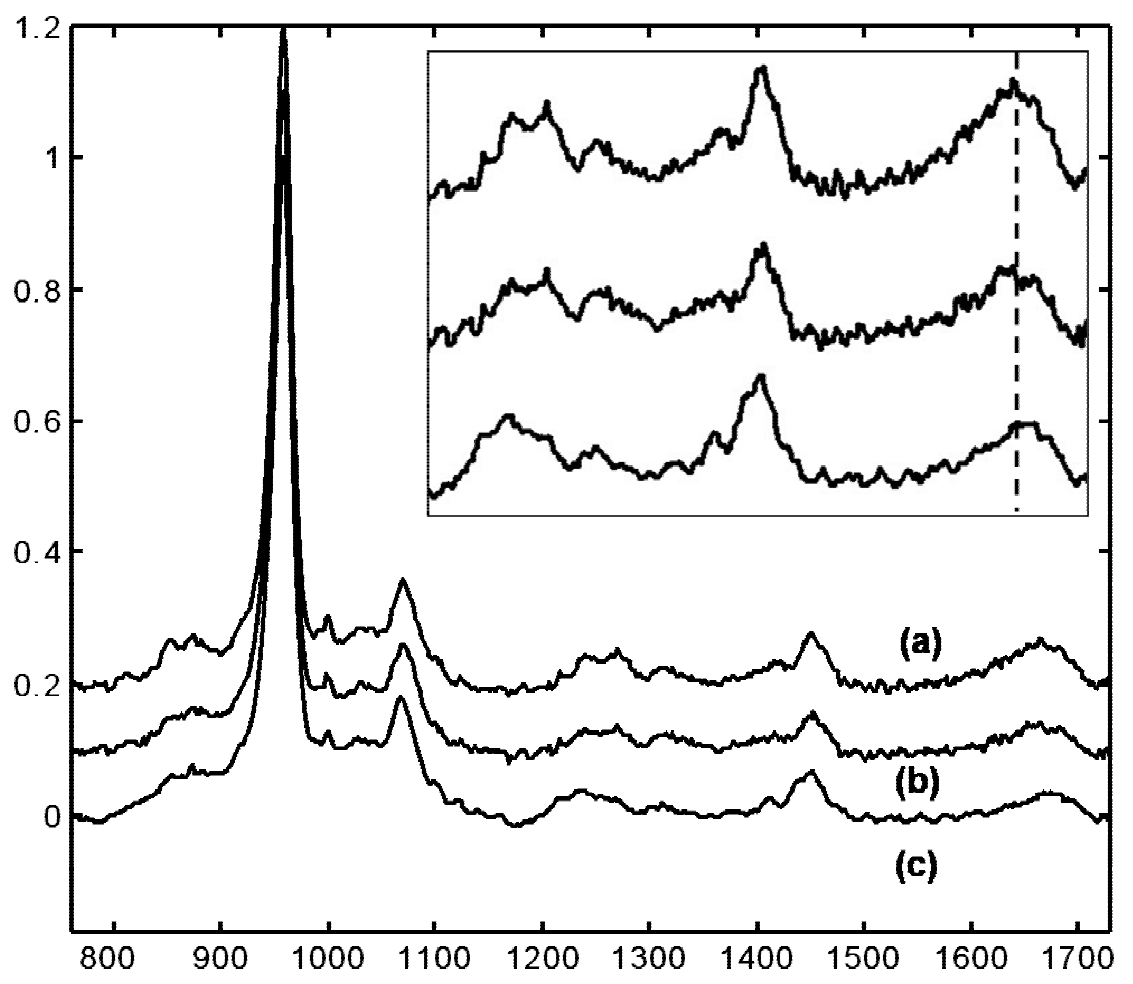

\section{Raman Shift (cm-1)}

Figure 2. Raman score image of Brtl mouse cortical bone tissue, double mutation. Contrast shows distribution of defect tissue.
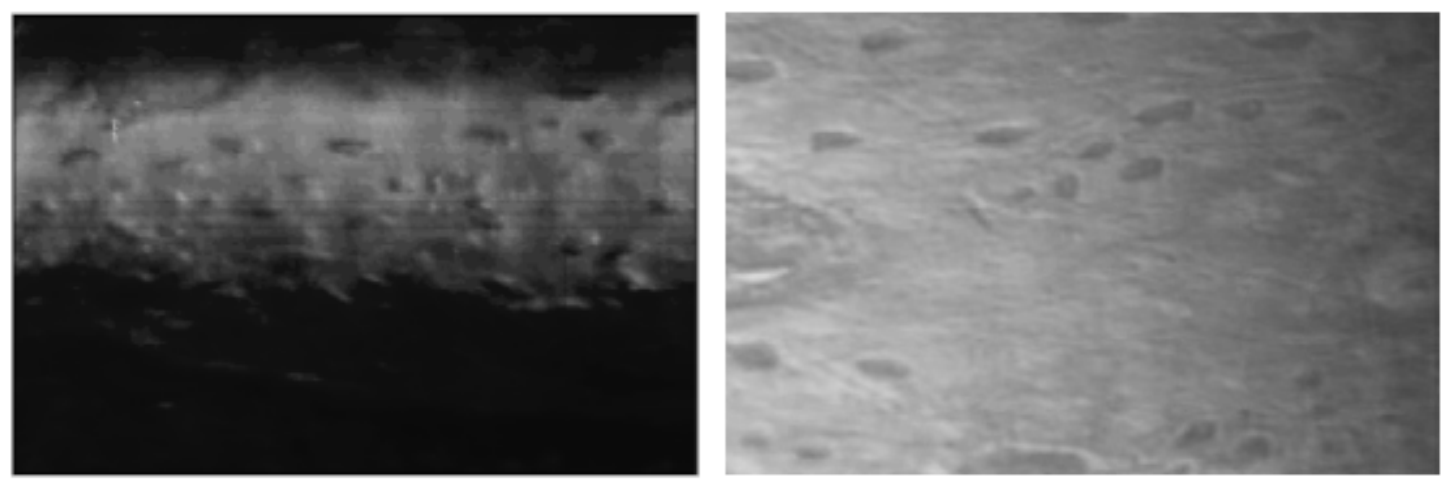